\title{
Analysis on the Curative Effect Observation of Endometrial Polyps' Resection under the Hysteroscopy
}

\author{
Xingyun Sun ${ }^{1, a}$, Yunxia Xue ${ }^{2, *}$
}

${ }^{1}$ Obstetrics and Gynecology clinic, The third People's Hospital of Jinan, Jinan, 250132, China

${ }^{2}$ Obstetrics and Gynecology, The third People's Hospital of Jinan, Jinan, 250132, China

axingyunsun@126.com

*Corresponding author yuxiaxue@126.com

\section{Keywords: Hysteroscopy; Endometrial polyps; Electric resection}

\begin{abstract}
Objective: To observe and analyze the curative effect of endometrial polyps' resection under the hysteroscopy. Methods: From March in 2012 to May in 2014, we select 64 examples of the intrauterine membrane polyp patients in our hospital, and who are randomly divided into two groups, 32 patients in each group. The treatment group uses the electric resection under the hysteroscopy surgery treatment, and the control group adopts the curettage under the hysteroscopy, to observe the curative effect of two groups. Results: The operation time of the treatment group is $(9.2 \pm 3.6) \mathrm{min}$ and the control group is $(9.3 \pm 4.1) \mathrm{min}$, and there is no significant difference. The treatment group patients interoperate blood loss is $(22.5 \pm 6.5) \mathrm{ml}$, and the control group is $(23.2 \pm$ $5.8) \mathrm{ml}$ and there is no significant difference, no statistical significance $(\mathrm{P}>0.05)$. The treatment group patients postoperative period of postoperative 6 months review is $(141.2 \pm 30.8) \mathrm{ml}$, and the control group 6 months postoperatively with postoperative review menstrual quantity is $(252.6 \pm 45.7) \mathrm{ml}$, which has the significant difference statistically significant, ovulation endometrial thickness and the recurrence rate compared with the control group, the differences is statistically significant $(\mathrm{P}<0.05)$. Conclusion: The endometrial polyps' resection under the Hysteroscopy can effectively improve the treatment effect, little injury and treatment process, and the late recovery is good. The recurrence rate is low after the treatment. This method is the common surgical method in the treatment of the endometrial polyps, and is worth clinical promotion.
\end{abstract}

\section{Introduction}

The endometrial polyps (endometrial polyps, EMP) is by the base layer of the film inside the uterus gland and hyperplasia, in single sex, colour and lustre is similar to that of a lining around. Foreign studies have found that the incidence of EMP less than 35 years old is about $3 \%$ and $>35$ years old is to $23 \%$, the incidence of postmenopausal women was $31 \%$, the peak onset age of 50 . The traditional diagnosis method of EMP relies mainly on the ultrasonic auxiliary examination and diagnostic curettage joint the examination to diagnosis. Hysteroscopy now makes it possible to look straight into the uterus of the application of hysteroscopy in the diagnosis of EMP is used widely. Endometrial polyps' treatment has the following several ways: hysteroscopy with cervical resection of endometrial polyps (TCRP) and TCRP plus postoperative adjuvant, TCRP + hysteroscopy with cervical endometrial resection, etc. At present domestic information about hysteroscopy treatment of endometrial polyp is less, there is no analysis of system comprehensive treatment of endometrial polyps. Selection in March 2012 to May 2014, our hospital 64 examples of intrauterine membrane polyp patients, surgery treatment, the curative effect is obvious, now report as follows.

\section{The Materials and Methods}

\section{The general information}

We select our hospital 64 examples of intrauterine membrane polyp patients from March 2012 to May 2014, and are randomly divided into two groups, 32 cases in each group, the treatment group of 25 to 50 years old, average age (33.8 \pm 4.2$)$; Course of 1-3 years, average duration of 2.1 
years; 20 patients with fertile period, 12 patients with menopause; The control group 24-49 years old, average age (32.6 \pm 5.3$)$; Course of 1-3 years, average duration of 2.0 years; 19 patients with menopause, menopausal 13 cases; Two groups of patients with no significant differences in age, illness, comparable.

\section{Methods}

Therapeutic group was treated with electric resection under hysteroscopy surgery, (1) the patient cut stone, regular disinfection vulva, vagina, put to have cervical dilation rods, wear sterile gloves, towel. Anesthesia can choose the intravenous anesthesia, lumbar hemp, continuous epidural anesthesia, if we can use the intubations anesthesia combined laparoscopic surgery. (2) when using ultrasound monitoring proper filling of bladder, clear display uterine body and bottom of the uterus. (3) with cervical clamp clamps the cervix, uterine cavity depth, dip guided by ultrasound number by expansion mouth to outer diameter greater than hysteroscopy surgery, usually for $10 \sim 11 \mathrm{~mm}$. (4) $5 \%$ glucose solution or 5\% liquid bulk palace, palace pressure setting in mean arterial pressure level. The electric current power is $80 \mathrm{~W}$, and the electric coagulation current is $60 \mathrm{~W}$ powers. (5) Under the ultrasound in mirror, check the polyp morphology, number, size, roots. (6) Remove a polyp from roots of lest recurrence in the future. When surface is rough vascular tissue, should first electric coagulation of blood vessels, and removing polyps. For multiple polyps can use after the removal of part of polyp negative pressure aspirator pump lining and polyp, coating on the surface of the polyp, lining is sucked, only interstitial tissue polyps, shrinkage, roots, easy to cut. (7) To examine the newspeak, reduce intrauterine pressure, electric coagulation homeostasis bleeder. (8) Measurement specimen weight, fixed, inspection. Control group using hysteroscopy under the curettage; and we observe the therapeutic effect of two groups of patients.

\section{Statistical processing}

With mathematical statistics software SPSS19.0 for data collection and statistical analysis, and chi-square test, $\mathrm{P}<0.05$, significant difference has statistical significance.

\section{The results}

\section{Two groups with the operation time and the intraoperative blood loss}

The operation time of the treatment group is $(9.2 \pm 3.6) \mathrm{min}$ and the control group is $(9.3 \pm 4.1)$ min, and there is no significant difference. The treatment group patients interoperate blood loss is $(22.5 \pm 6.5) \mathrm{ml}$, and the control group is $(23.2 \pm 5.8) \mathrm{ml}$ and there is no significant difference, no statistical significance $(\mathrm{P}>0.05)$. As shown in table 1 .

Table 1: Compared two groups of the operation time and the intraoperative blood loss

\begin{tabular}{cccc}
\hline Group & $\mathrm{n}$ & $\begin{array}{c}\text { The operation } \\
\text { time }(\mathrm{min})\end{array}$ & $\begin{array}{c}\text { The interoperate } \\
\text { blood loss }(\mathrm{ml})\end{array}$ \\
\hline The treatment group & 32 & $9.2 \pm 3.6$ & $22.5 \pm 6.5$ \\
The control group & 32 & $9.3 \pm 4.1$ & $23.2 \pm 5.8$ \\
$\chi 2$ & & 0.065 & 0.143 \\
P value & & $P>0.05$ & $P>0.05$ \\
\hline
\end{tabular}

\section{Two groups of the postoperative menstrual quantity}

The treatment group patients with postoperative period of postoperative 6 months review is $(141.2 \pm 30.8) \mathrm{ml}$, and the control group 6 months postoperatively in patients with postoperative review menstrual quantity is $(252.6 \pm 45.7) \mathrm{ml}$, and compared with significant difference statistically significant, ovulation endometrial thickness and the recurrence rate compared with the control group, significant difference statistically significant $(\mathrm{P}<0.05)$. See Table 2. 
Table 2: Two groups of the postoperative menstrual quantity

\begin{tabular}{ccc}
\hline Group & $\mathrm{n}$ & $\begin{array}{c}\text { The postoperative menstrual } \\
\text { quantity }(\mathrm{ml})\end{array}$ \\
\hline The treatment group & 32 & $141.2 \pm 30.8$ \\
The control group & 32 & $252.6 \pm 45.7$ \\
$\chi 2$ & & 10.253 \\
P value & $<0.05$ \\
\hline
\end{tabular}

\section{The endometrial polyps' resection before and after menstruation of the treatment group}

By hysteroscopy endometrial polyps resection under the contrast before and after menstruation, before resection by the volume score and menstrual period respectively are (265.28 \pm 71.67$)$ and (7.6 $\pm 2.9) \mathrm{d}$, after removal of the amount of grading and menstrual period, respectively are $(22.65 \pm 10.97)$ and $(3.9 \pm 0.2) \mathrm{d}$, obvious difference has statistical significance before and after treatment, $\mathrm{p}<0.05)$. See Table 3.

Table 3: The endometrial polyps' resection before and after menstruation of the treatment group

\begin{tabular}{cccc}
\hline Group & $\mathrm{n}$ & The volume score & $\begin{array}{c}\text { The menstrual } \\
\text { period }\end{array}$ \\
\hline $\begin{array}{c}\text { Before } \\
\text { menstruation }\end{array}$ & 32 & $265.28 \pm 71.67$ & $7.6 \pm 2.9$ \\
After & 32 & $22.65 \pm 10.97$ & $3.9 \pm 0.2$ \\
menstruation & & 15.328 & 8.065 \\
$\chi 2$ & $<0.05$ & $<0.05$ \\
P value & & & \\
\hline
\end{tabular}

\section{Discussion}

The endometrial polyps is a kind of mesenchymal cells cloned hyperplasia, accompanied by the tumor glands composition of benign tumors, is one of the most common cause of abnormal uterus bleeding lesions. Due to its easy to hide (type I and type II ) endometrial carcinoma and precancerous lesions, so once found that should be removed promptly and all drew microscopy in detail. Endometrial polyps in patients with abnormal uterine bleeding symptoms in the early diagnosed and given priority to with surgical treatment, also make the treatment has good results, but the late poor morphology and cell differentiation of endometrial polyps, in addition to surgical treatment, often also need other adjuvant therapy, including radiation therapy, chemotherapy, or hormone therapy and other methods. For the disease, in addition to prevent the happening of the it, our strategy is to early diagnosis and early treatment, but for the late and less cell type patients the treatment way and the result has been a thorny problem. So there is a lot of research on cancer metastasis and invasion mechanism. Endometrial polyps can occur in any of childbearing age and postmenopausal age, the average age of about 38 years old. There are some situations may be related to the occurrence of endometrial polyps, now think of female hormones (Estrogen) stimulation is excessively endometrial hyperplasia or endometrial polyps most basic factors. Studies have showed that compound oral contraceptives have lower risk than simple. Most of the patients with endometrial polyps can have abnormal vaginal bleeding especially postmenopausal bleeding and menopausal symptoms of abnormal bleeding. The diagnosis of endometrial polyps is mainly by sectional uterine enlargement surgery. In addition they are helpful to the diagnosis of endometrial polyps, but to establish the diagnosis of uterine or want to rely on sectional expansion technique of histopathology confirmed. The prognosis of endometrial polyps and cancer cells form, the degree of 
differentiation, the invasion depth, or the peritoneal washing fluid cytology, ovary and oviduct was invaded, presence of lymph node metastasis, or the transfer of other organs and so on.

Endometrial polyps can happen at any age after puberty, but no clinical symptoms or abnormal uterine bleeding and infertility, medication is often ineffective. Always the traditional treatment is initiated and curettage in the diagnosis treatment, total hysterectomy for conservative treatment is invalid. In accordance with the requirements of different age, different fertility line hysteroscopy surgery may retain the womb, retain reproductive function, and become the minimally invasive surgery treatment of endometrial polyp.

In patients with abnormal uterine bleeding caused by endometrial polyps disease takes the second place, second only to endometrial hyperplasia. The cause may be related to inflammatory disorders, endocrine disorders, especially high estrogen levels. Most scholars believe that from immature endometrial polyp endometrial estrogen levels at different positions of the different, cause of the estrogen receptor effect difference, so that local hyperplasia of excessive and form polyps, the surrounding other lining is often shown as polyploidy hyperplasia. For patients with abnormal uterine bleeding, must line of endometrial pathologic examination, eliminate polyp progression and endometrial cancer. For patients with no fertility requirements, if simply removing polyps only solved the partial hyperplasia, around other abnormal hyperplasia of will continue to grow, again polyps or more serious endometrial hyperplasia. Therefore, the patients without fertility requirements, at the same time of polyps' resection lining, can prevent the polyp recurrence. Endometrial electricity cut method to remove the diseased tissue, hyperplasia of the most no longer, polyp recurrence, no longer keep the patient's uterus, maintain the normal anatomy of the pelvic floor, can be used as alternative of total hysterectomy minimally invasive surgical method for the treatment of endometrial polyp.

The literature reports claim to have fertility polyp patient take the hysteroscopy polyp resection or curettage after hysteroscopy locate, two groups of surgical method the postoperative curative effect comparison of the hysteroscopy was lower than those of polyp recurrence of curettage, recurrence time later. At the same time, the cause of the polyp associated with estrogen single stimulus for a long time, so the patient also can use of oral and the hysteroscopy surgery for the treatment of endometrial polyps treatments. For older, vaginal bleeding and no more bear the requirements of the patients with endometrial polyp, more access to removal of the film inside the uterus, endometrial resection, electric coagulation ball electrode to the membrane technique, laser to lining, hot ball to endometrial lining and microwave technique, etc., can reduce the polyp recurrence, reduce menstrual quantity, operation effectiveness $100 \%$, almost no recurrence. In this study, it is according to the actual situation of the four different hysteroscopy surgery treatments.

The endometrial polyps can happen at any age after puberty, develops in per menopausal or postmenopausal, exact unknown etiology, uterine cavity operation may be related to local, local inflammation and endocrine disorder. Endometrial polyps can think in a previous study menopause local high levels of estrogen and progesterone receptors and membrane lower expression or (and) high estrogen receptor expression, cell proliferation and apoptosis imbalance, cancer gene mutation and so on. Endometrial proliferation on estrogen only when menopause reaction, lack of progesterone receptor, or progesterone, and lead to endometrial hyperplasia polyps or polyploidy change gradually, internal environment changes may continue to proliferate and produce. The endometrial polyps of department of gynecology often no abnormal findings, easily misdiagnosed as dysfunctional uterine bleeding. If endometrial polyps protrudes from the cervix, can see or touch is easy to diagnose, but must and the cervical polyp. Ultrasound is one of the commonly used auxiliary examination of department of gynecology, endometrial polyps is endometrial glands and hyperplasia of fibrous limitations uplift and formation of a pedicle of the tumor lesion, and endometrial polyps ultrasound mainly for abnormal intrauterine echo clumps or endometrial thickening.

For FMP treatment, drug therapy, serious and even removal of the uterus. Traditional treatment scheme has certain limitations, such as simple diagnostic curettage has certain blindness, scraping or excessive leakage and curettage, etc. And hysteroscopy to diagnosis and treatment is currently 
the only instrument can look straight into the uterus. After the hysteroscopy treatment of the FMP hysteroscopy procedures were positioning to remove polyps or removing polyps under hysteroscopy to look straight. According to the requirements of the patients with different age in our hospital, there is different birth to select different hysteroscopy surgery. Generally for postmenopausal women, because of its easy without symptoms polyps, once in a while to find the characteristics of it once found, should actively treatment; For patients with no fertility requirements, at the same time of polyps resection lining, can prevent the polyp recurrence; For childbearing age have fertility demander, joint pregnant again after hysteroscopy thoroughly remove polyps hormonal state drug treatment. Under hysteroscopy surgery in the treatment of endometrial polyp of the biggest advantage is minimally invasive, and polyp in direct from the roots of all, the complete excision without affecting the rest of the normal endometrium, reduces its recurrence rate. Moreover hysteroscopy with shorter operation time, less bleeding, faster recovery, high retained viscera function, patient satisfaction, makes the hysteroscopy become the gold standard for diagnosis and treatment of endometrial polyp. This study of FMP patients of our hospital was treated with hysteroscopy surgery, results show that the intraoperative and postoperative complications, surgical success rate of $100 \%$. Postoperative follow-up is 3 to 24 months, no recurrence. With hysteroscopy in clinical application, greatly improving the diagnosis rate of FMP, also significantly reduce the missed diagnosis especially in observing intrauterine small lesions and malignant lesions were the protrusions more meaningful, this method is worthy of clinical application.

\section{Conclusion}

The hysteroscopy surgery is the best way for the treatment of endometrial polyps, especially for the coarse of endometrial polyps. If it is the pure and curettage, it is easy to miss endometrial polyps, or can't shave their polyps roots and easily to relapse. The hysteroscopy can adopt the way of cutting removal of coarse endometrial polyps; the treatment effect is improved obviously. The operation scope is limitation; the exact is effect, less bleeding, shorter operation time, and rapid recovery. This group of data shows that the endometrial polyps' resection under the hysteroscopy can effectively improve the treatment effect, and the late recovery is good. The recurrence rate is low after the treatment. This method is the common surgical method in the treatment of the endometrial polyps, and is worth clinical promotion.

\section{Acknowledgement}

This research was financially supported by the National Science Foundation.

\section{References}

[1] MARITLIENG,OLAVISTRE,ERIKQVIGSTAD. Treatment of endometrial polyps: a systematic review[J]. Acta Obstetricia et Gynecologica Scandinavica. 2012 (8)

[2] Jian-Hua Wang,Jin Zhao, Jun Lin. Opportunities and Risk Factors for Premalignant and Malignant Transformation of Endometrial Polyps: Management Strategies[J]. The Journal of Minimally Invasive Gynecology. 2012 (1)

[3] Tamar Perri, Kurosh Rahimi, Agnihotram V. Ramanakumar, Karen Wou,Dragana Pilavdzic, Eduardo L. Franco, Walter H. Gotlieb,Alex Ferenczy. Are endometrial polyps true cancer precursors?[J]. American Journal of Obstetrics and Gynecology. 2013 (3)

[4] Ayhan Gul, Mustafa Ugur, Cantekin Iskender, Ebru Zulfikaroglu, Gulnur Ozaksit. Immunohistochemical expression of estrogen and progesterone receptors in endometrial polyps and its relationship to clinical parameters[J]. Archives of Gynecology and Obstetrics. 2012(3)

[5] Luigi Nappi,Ugo Indraccolo,Attilio Di Spiezio Sardo,Giorgio Gentile,Katia Palombino,Maria Antonietta Castaldi,Marialuigia Spinelli,Pantaleo Greco. Are Diabetes, Hypertension, and Obesity 
Independent Risk Factors for Endometrial Polyps?[J]. The Journal of Minimally Invasive Gynecology. 2012 (2)

[6] Gabriela Baiocchi, Natalina Manci,Michela Pazzaglia, Laura Giannone,Liliana Burnelli, Ettore Giannone, Daniela Fratini,Gian Carlo Di Renzo. Malignancy in endometrial polyps: a 12-year experience[J]. American Journal of Obstetrics and Gynecology. 2009 (5)

[7] O. Gregoriou,S. Konidaris,N. Vrachnis, K. Bakalianou, N. Salakos,K. Papadias,A. Kondi-Pafiti,G. Creatsas. Clinical parameters linked with malignancy in endometrial polyps[J]. Climacteric. 2013(5)

[8] Elizabeth Taylor,Victor Gomel. The uterus and fertility[J]. Fertility and Sterility. 2013(1)

[9] Federica Scrimin,Uri Wiesenfeld, Alberto Candiotto, Stefania Inglese, Luca Ronfani,Secondo Guaschino. Resectoscopic treatment of atypical endometrial polyps in fertile women[J]. American Journal of Obstetrics and Gynecology. 2013(4)

[10] Reginaldo Guedes C. Lopes, Edmund Chada Baracat, Luiz Cavalcanti de Albuquerque Neto,José Francisco Dória Ramos, Salete Yatabe,Daniela Baptista Depesr,Umberto Gazi Lippi. Analysis of estrogen- and progesterone-receptor expression in endometrial polyps[J]. The Journal of Minimally Invasive Gynecology . 2013 (3)

[11] Chavez NF,Gamer EO,Khan W, et al. Does the introduction of newtechnology change population demographics? Minimally invasivetechnologies and endometrial polyps. Gynecologic and Obstetric Investigation . 2012(3)

[12] Bakour SH,Gupta JK,Khan KS. Risk factors associated with endometrial polyps in abnomal uterine bleeding. International Journal of Gynecology and Obstetrics . 2013(2) 